\title{
Percutaneous Coronary Interventions over the last three decades: the adjunctive devices (role, development and perspectives)
}

\section{Leo Finci}

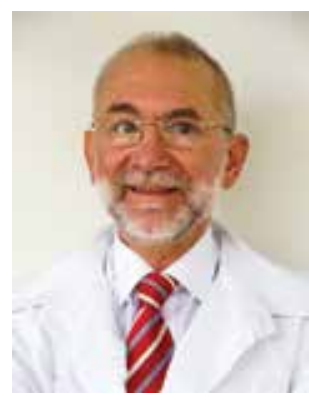

\author{
Dr Leo Finci \\ Membre de la Société Suisse de Cardiologie, \\ Fellow of the American College of Cardiology, \\ Founding Fellow of the European Society of Cardiology.
}

Abstract

Percutaneous coronary interventions ( $\mathrm{PCl}$ ) performed on large-scale basis in routine practice with documented long-term clinical benefit for the patient are balloon angioplasty introduced in 1977 and coronary stenting since 1986. Other coronary devices, so-called "adjunctive", have triggered a great admiration at the time of its initial clinical application but showed to be less successful at the long-term follow-up. However, many adjunctive devices are still necessary in 1-2\% of all PCl procedures for complex cases. This article is a short, comprehensive review retracing the conception and destiny of these devices. Coronary balloon catheters, a standard device with advance "therapeutic" features comprise: cutting, drug eluting and high pressure non-compliant balloon catheters. Atherectomy devices can be directional for lesions "debulking", rotational (Rotablator) for calcified, long lesions and recently Orbitral supposed to have less no-reflow phenomenon. Excimer Laser angioplasty for treatment of "uncrossable" lesion was claimed much in the past. Brachytherapy is used almost exclusively for in-stent restenosis. A numerous thrombectomy and embolic protection devices exist but none has shown a proven clinical benefit in randomized studies. With an advancement in medical technology adjunctive devices will play more important role in the future, especially for recanalization of chronic total occlusion and during acute myocardial infarction.

Key words percutaneous coronary interventions, stents, adjunctive devices.

\section{Introduction}

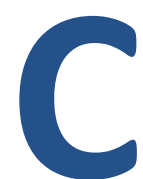

oronary balloon angioplasty (PTCA) introduced in 1977, followed by coronary stenting in 1986 represent the only two percutaneous interventions $(\mathrm{PCl})$ that have documented and confirmed clinical benefit for the patient and are being performed in current practice at the large-scale basis ${ }^{1}$. Many other coronary devices, still remaining as a part of interventional cardiologist's arsenal, have triggered a great admiration at the time of initial clinical application but turned out to be less successful at the long- term follow-up. The author of this article has been in the field of interventional cardiology from the begging of its routine application and believes that these so-called "adjunctive" devices need to be mentioned. It is like reading a medical paper that shows negative result - but from it, one can learn a lot, sometimes even more than reading a study with positive, significant result. During last three decades, the level of performed $\mathrm{PCl}$ has progressively increased reaching currently a steady usage level while new device technologies are still in progress (Figure 1). For this analysis, Switzerland was chosen as sample country for comparisons due to its stable politi- 


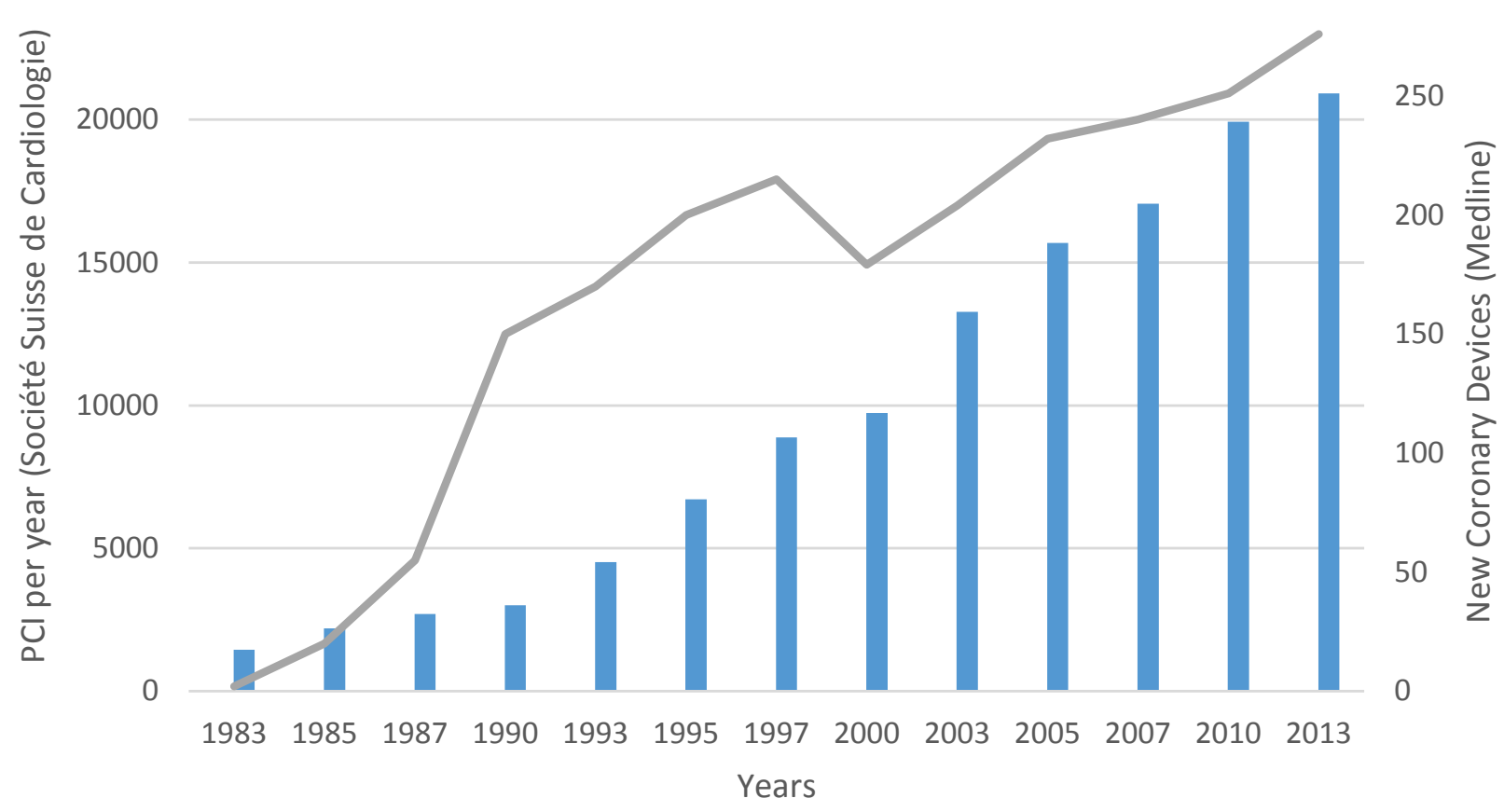

Figure 1. Correlation of $\mathrm{PCl}$ performed in Switzerland with publications on new coronary devices (number per year) during last three decades

cal and medical situation that had no variations over the examined time period, unlike the rest of the Europe, where creation of the European Union, reunification of Germany and other variables made an impact on the interventional cardiology statistics. New coronary devices were analyzed through the PubMed-Medline research coupled with clinical experience. Furthermore, over the last several years, more attention has been paid by the international cardiology community to their organized development and introduction of special events taking place in the course of congresses such as the Innovation session during the European congress of Cardiology ${ }^{2}$. The purpose of this article is to make a short comprehensive review of the conception and destiny of adjunctive devices. Coronary devices are classified according to their mechanism of action and none of the devices that are used for pure diagnostic purpose (e.g. intravascular ultrasound) is mentioned.

\section{Balloon catheters}

The idea to perform more interventions inside the coronary vessel using a simple balloon catheter with advance "therapeutic" features is still very accurate. Since 1992 "cutting" balloons have been developed to challenge complex lesions resistant to conventional balloon angioplasty ${ }^{3}$. Different types exist, using either single or multiple microsurgical blades bonded longitudinally to its surface, suitable for creating discrete longitudinal incisions in the targeted atherosclerotic coronary segment which are being activated during balloon inflations. Its current use is for "high-pressure balloon resistant lesions" including de novo lesions and in-stent restenosis (AngioSculptTM Scoring Balloon, Angioscore, USA). However, compared to PTCA there were no significant differences at 6-month follow-up in angiographic and clinical results. The high-pressure noncompliant balloons that can tolerate the pressure of 40 bars are now available (OPN NC ${ }^{\circledR}$, high pressure balloon catheter, SIS Medical AG, Winterthur, Switzerland). Drug eluting balloon (DCB) combines features of active drug and balloon inflation. The low rates of adverse clinical outcomes makes DCB a feasible treatment option in small vessel coronary artery disease, diffuse disease and bifurcation lesions. Their superiority has been proven only for the indication of the in-stent re-stenosis, as shown in a recent meta-analysis ${ }^{4}$, where the need for TLR was significantly reduced compared to other treatments. As a novel strategy, DCB still has a long way to ride before its full implementation. The only commercially available drug for DCB is Paclitaxel.

\section{Atherectomy}

There are three different types of atherectomies: $\mathrm{Di}$ rectional (DCA), Rotational (RA) and recently introduced Orbital Atherectomy. The DCA is probably the greatest deception of all coronary devices. Developed by John Simpson in 1984, just a few year after clinical introduction of his movable coronary guide-wire, DCA was supposed to decrease the high restenosis rate observed after PTCA (30-50\%). The device, called AtheroCath ${ }^{\mathrm{TM}}$ (Abbott Vascular, Redwood City, CA, USA) consists of a cylindrical metal housing with a lateral side window cutter and a low pressure balloon on the contralateral side. Low pressure balloon inflation assures for proper tissue-cutter apposition. 
The plaque is gently displaced into the window housing and "shaved-off", using the cutter, into the collecting nose cone at the distal tip of the device. In an early multi-center registry, DCA was successful in $85 \%$ of cases, and as such was subsequently comparable to success in balloon angioplasty. After having been approved by FDA, it gained confidence worldwide as an alternative to balloon angioplasty, and by 1990's accounted for approximately $10 \%$ of $\mathrm{PCl}$ procedures in the United States. In the following years several non-randomized studies showed an improved intraluminal coronary gain after DCA as compared to PTCA that generated an extreme enthusiasm for the device. However, in 1993 after publication of a randomized CAVEAT study showing higher restenosis (57\% versus $50 \%$ ) and complication rates (11\% versus $5 \%$ ) with DCA compared to PTCA, the use of DCA rapidly decreased. The protagonists of atherectomy ${ }^{5}$ welcomed the new multicenter USA clinical trial with Orbital atherectomy device on 443 consecutive patients with severely calcified coronary lesions ${ }^{6}$.

Rotablator TM (Boston Scientific USA) is the most known device of RA. It can effectively ablate calcified plaques, facilitating stent delivery and expansion. Practice guidelines recommend its use for preparation of heavily calcified or severely fibrotic lesion that cannot be crossed by a balloon or adequately dilated. However, late restenosis remains high when it is used as a stand-alone therapy or with bare-metal stents. Some observational study suggested a favorable long-term results of RA followed by DES implantation, but a randomized study including 240 patients showed that routine lesion preparation using RA did not reduce late lumen loss even after implantation of DES at 9 months follow-up ${ }^{7}$.

\section{Laser}

The potential advantages of intracoronary laser angioplasty are to ablate the plaque material and vaporize all atherosclerotic plaque along the arterial wall. The bulk removal of plaque material could improve acute procedural success rates, decrease complication rates, take care of "untreatable" lesions, and decrease restenosis rates. Several types of lasers have been used in the past (Argon, Holmium), but only the excimer laser is still in practice (ELCA ${ }^{\circledR}$ Laser Ablation Catheter, Spectranetics USA). The coronary laser catheters are offered in sizes ranging from 0.9 to $2.0 \mathrm{~mm}$ in diameter and contain up to 250 small, flexible optical fibers mounted within a thin plastic tube.

The great enthusiasm to use the laser angioplasty for the treatment of coronary occlusions was raised in 1986 because laser energy can vaporize atherosclerotic plaque, and there may be no requirement for a pre-existing channel. However, high rate of complications, thermal injury and collateral miss have limited its application. A systemic literature search ${ }^{8}$ performed in 2014 by McGill University in Canada, found no benefit even in patients with un-crossable coronary lesion. Occurrence of complications such as coronary dissection (up to $9 \%$ ), myocardial infarction (0-10\%), or major bleeding (0-6\%) and increased procedure cost influenced the decision not to recommend its use in Canada.

\section{Brachytherapy}

The radiation is believed to inhibit the cellular proliferation. Radiation for treatment of in-stent restenosis has been using two sources: gamma and beta radiation. Currently approved are the Checkmate System (Cordis Corporation) that uses gamma radiation and the BetaCath System (Novoste Corporation) that uses beta radiation. Approval by the FDA in USA for both of these devices is limited their use in stents that had been implanted in the past, and then re-stenosed. In the years following 2000, the treatment of restenosis after bare metal stent implantation using brachytherapy raised a great hope, but rapid developments in drug eluting stents had progressively pushed the brachytherapy a side. A meta-analysis ${ }^{9}$ in 2011 of 12 studies and comparing the outcomes of drug-eluting stents versus intracoronary brachytherapy for in-stent restenosis suggests that the use of drug-eluting stents for in-stent restenosis is associated with reduced occurrences of target-vessel revascularization and binary restenosis. The American College of Cardiology Guidelines (2011) do not recommend brachytherapy for the prevention of restenosis as the lower rates of restenosis occur with the use of drugeluting stents in comparison to bare metal stents or vascular brachytherapy. Many technical limitations are present within brachytherapy, such as the operator's protection against gamma radiation, coronary lesion geographical miss (unwilling exposure of healthy tissue to the radiation) and the absence of distinguished longterm benefit.

\section{Thrombectomy}

A numerous devices with different mechanisms of action exist all having the same objective to reduce distal thrombus embolization and improve myocardial perfusion. In a meta-analysis there was a significant improvement in ST-segment resolution, myocardial blush and TIMI grade 3flow as parameters of myocardial perfusion, as well as clinical parameters such as reduction in mortality. Non-manual, mechanical thrombectomy may have a role in selected patients with large caliber vessels and heavy thrombus burden ${ }^{10}$.

Catheter aspiration thrombectomy uses a catheter that is advanced over a guidewire to the thrombus whereby syringe suction is used to aspirate the debris. Devices used for this procedure include the DiverTM, Export $^{\circledR}$, ProntoTM, RescueTM, Thrombuster $^{\circledR}$, and TransVascular Aspiration Catheter ${ }^{\circledR}$. Mechanical thrombectomy devices apply energy directed through 
saline jets or a rotating catheter head to facilitate breakup of the thrombus prior to its active aspiration. These devices include the AngioJet ${ }^{\circledR}$ and $\mathrm{X}$-Sizer ${ }^{\circledR}$.

\section{Embolic protection}

Embolic protection devices can be proximal and distal, using either balloon or filters. Distal device employs an occlusion balloon advanced over a guide-wire distal to the thrombus in order to trap and aspirate thrombotic debris released during angioplasty and stenting procedures such as the PercuSurge GuardWire ${ }^{\circledR}$, FilterWire EXTM, SpideRXTM, AngioGuardTM and Filtrap. A study concerning these devices conducted in 2011 by the USA Agency for Healthcare Research and Quality examined 53 randomized trials $(n=8,185)$ and 9 observational studies $(n=1,479)$ and found no significant impact on mortality, myocardial infarction, stroke, or MACE versus a control when using the longest duration of follow-up ${ }^{11}$.

\section{Stents}

There are several so called "dedicated" stents, designed for specific lesion categories such as bifurcations or vein grafts, which are on the market. None of them has showed clinical benefit as compared to the standard technique ${ }^{12}$. Covered stent are of real utility in case of coronary artery perforation such as the Grafmaster ${ }^{\circledR} \mathrm{RX}$, Abott, and mPK Papyrus, Biotronik, Switzerland - a more flexible stent. Specially designed drug eluting stents capable to accommodate multiple drugs in a special reservoirs incited a great hope at the beginning. The example is the NEVO ${ }^{\circledR}$ Cordis, USA, coronary stent but it was retrieved from the market after Johnson \& Johnson Company announced halt of their research activities in interventional cardiology. The MGuard (Inspire MD, Boston USA) stent utilizes MicroNet ${ }^{\mathrm{TM}}$ technology, which is a circular knitted mesh that wraps around the stent to protect patients from plaque debris flowing downstream upon deployment (during acute myocardial infarction). Bioresorbable vascular scaffolds (BVS) are very promising devices and will certainly expand in the future ${ }^{13}$.

\section{CTO}

Recanalization of Chronic total coronary occlusions (СТО) has been for years and is still now a real battlefield for use of adjunctive devices (Figure 2). A recent metaanalysis ${ }^{14}$ study including 18,061 patients showed an angiographic success rate of $77 \%$ with following complications: contrast nephropathy $3.8 \%$, coronary perforation $2.9 \%$, myocardial infarction $2.5 \%$, death $0.2 \%$, emergent coronary surgery $0.1 \%$, tamponade $0.3 \%$ and stroke $0.01 \%$.

The concept of mechanical recanalization with consequent angioplasty is still applied with different possibilities such as sub intimal tracking, re-entry technique or retrograde approach. Micro catheters represent a real

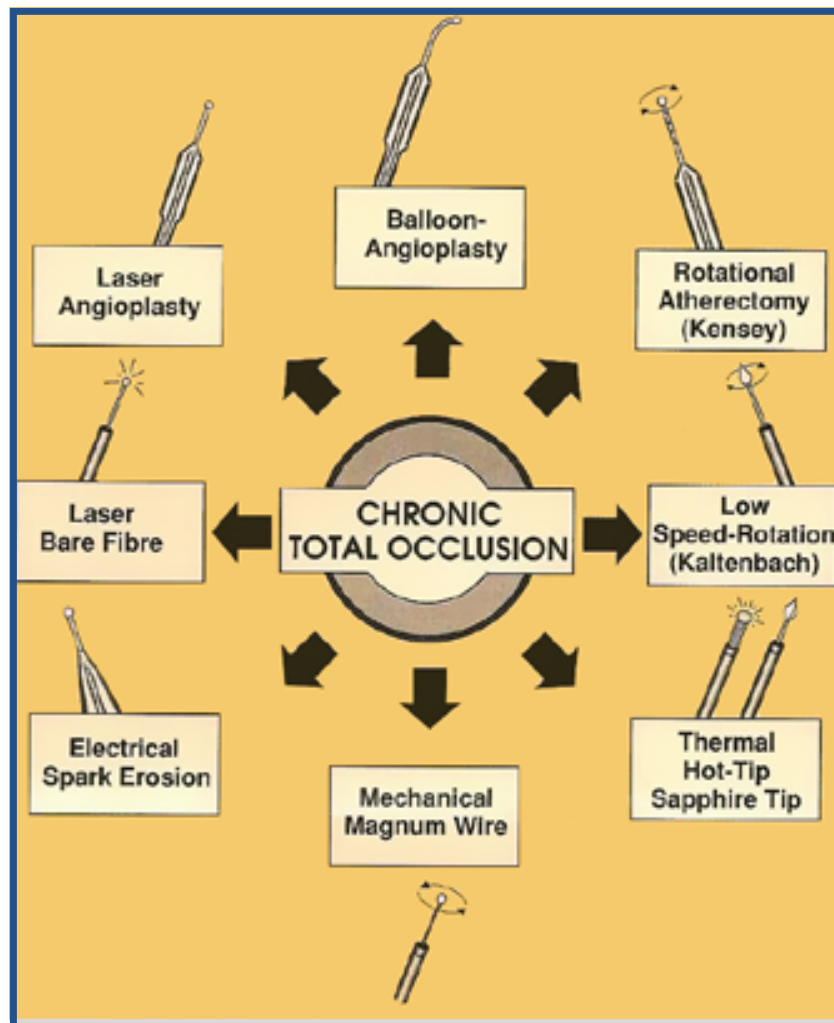

Figure 2. Adjunctive devices for CTO as proposed in the year 1990

advancement, the most known are $\operatorname{Transit}^{\circledR}$ (Cordis, Miami, United States), Finecross ${ }^{\circledR}$, Pro Great ${ }^{\circledR}$ (Terumo, Japan). They have the advantage of large lumen and trackability for selective injection and tortuosity, whereas Corsair ${ }^{\circledR}$ (Asahi Intecc, Aichi, Japan) has more support, even beyond a calcified or tortuous segment. The Tornus device (Asahi) is a catheter made of 8 stainless steel strands woven together to enhance flexibility and strength in exchanging wires, delivering balloons and providing support for CTO procedures. The Frontrunner (LuMend, Cordis, USA) device is designed to create intraluminal blunt micro dissection to facilitate penetration of the fibrous cap. Two other recent systems for lesion crossing and lumen re-entry technologies include CrossBoss catheter (BridgePoint Medical, Plymouth, Minnesota), a metal micro catheter with a rounded tip that can advance through a CTO eventually into the sub intimal space. Then Stingray balloon and Stingray guide wire systems (BridgePoint Medical) which are employed to penetrate the distal true lumen for re-entry. In a catheterization laboratory that performs these interventions, other adjunctive safety devices are necessary like: covered stents, thrombectomy devices, snares, embolization coils and delivery systems to manage possible complications. With the advancement of medical technology, adjunctive devices will play more important role in the future, especially for recanalization of chronic total occlusion and for patients with acute coronary syndrome undergoing percutaneous coronary intervention. 


\section{References}

1. Laslett LJ, Alagona P Jr, Clark BA, Drozda JP Jr, Saldivar F, Wilson SR, Poe C, Hart M. The worldwide environment of cardiovascular disease: prevalence, diagnosis, therapy, and policy issues: a report from the American College of Cardiology. J Am Coll Cardiol. 2012; 60(25 Suppl):S 1-49.

2. http://www.escardio.org/congresses/esc-2015/call-science/ Pages/welcome.aspx.

3. Michael SL, Varinder S, Thomas JN, James RW. Review: cutting balloon angioplasty. J Invasive Cardiol. 2002;14 (9). http://www. medscape.com/viewarticle/442312.

4. Piccolo R, Galasso G, Piscione F, Esposito G, Trimarco B, Dangas $\mathrm{GD}$, Mehran R. Meta-analysis of randomized trials comparing the effectiveness of different strategies for the treatment of drugeluting stent restenosis. Am J Cardiol. 2014;114(9):1339-46.

5. Colombo A, Panoulas VF. After 3 decades, at long last, a new device to deal with calcific lesions. JACC Cardiovasc Interv. 2014;7(5):519-20.

6. Chambers JW, Feldman RL, Himmelstein SI, Bhatheja R, Villa AE, Strickman NE Shlofmitz RA, Dulas DD, Arab D, Khanna PK, Lee AC, Ghali MG, Shah RR, Davis TP, Kim CY, Tai Z, Patel KC, Puma JA, Makam P, Bertolet BD, Nseir GY. Pivotal trial to evaluate the safety and efficacy of the orbital atherectomy system in treating de novo, severely calcified coronary lesions (ORBIT II). JACC Cardiovasc Interv. 2014;7(5):510-8.

7. Abdel-Wahab M, Richardt G, Joachim Büttner $H$, Toelg R, Geist V, Meinertz T, Schofer J, King L, Neumann FJ, Khattab AA. Highspeed rotational atherectomy before paclitaxel-eluting stent implantation in complex calcified coronary lesions: the randomized ROTAXUS (Rotational Atherectomy Prior to Taxus Stent Treatment for Complex Native Coronary Artery Disease) trial. JACC Cardiovasc Interv. 2013;6(1):10-9.
8. Sinclair A., Dendukuri N. Excimer laser atherectomy for uncrossable coronary lesions and improperly deployed coronary stents. Montreal (Canada): Technology Assessment Unit (TAU) of the McGill University Health Centre (MUHC) 2014; Report no. 75. 28 p. Available at http://www.mcgill.ca/tau/files/tau/muhc_ tau_2014_75_excimer_b.pdf.

9. Lu YG, Chen YM, Li L, Zhao RZ, Fu CH, Yan H. Drug-eluting stents versus intracoronary brachytherapy for in-stent restenosis: a meta-analysis. Clin Cardiol. 2011 ; 34(6):344-51.

10. Costopoulos C, Gorog DA, Di Mario C, Kukreja N. Use of thrombectomy devices in primary percutaneous coronary intervention: a systematic review and Meta-analysis. Int J Cardiol. 2013 Mar 10;163(3):229-41.

11. Sobieraj DM, White CM, Kluger J, et al. Adjunctive devices for patients with acute coronary syndrome undergoing percutaneous coronary intervention. Comparative effectiveness review No. 42 Rockville, MD: Agency for Healthcare Research and Quality 2011; AHRQ Publication No. 11(12)-EHC089-EF. Available at www.effectivehealthcare.ahrq.gov/thrombusacs.cfm.

12. Généreux $P$, Kumsars I, Lesiak $M$, Kini $A$, Fontos $G$, Slagboom $T$, Ungi I, Metzger DC, Wykrzykowska JJ, Stella PR, Bartorelli AL, Fearon WF, Lefèvre T, Feldman RL, LaSalle L, Francese DP, Onuma $Y$, Grundeken MJ, Garcia-Garcia HM, Laak LL, Cutlip DE, Kaplan AV, Serruys PW, Leon MB. A randomized trial of a dedicated bifurcation stent versus provisional stenting in the treatment of coronary bifurcation lesions. J Am Coll Cardiol. 2015; 65(6):533-43.

13. Di Mario C, Caiazzo G. Biodegradable stents: the golden future of angioplasty? Lancet 2015; II vol 384:10-12.

14. Patel VG, Brayton KM, Tamayo A, Mogabgad O, Michael T, Lo N, Alomar M, Shorrock D, Cipher D, Abdullah S, Banerjee S, Brilakis E. Angiographic Success and Procedural Complications in $\mathrm{Pa}$ tients Undergoing Percutaneous Coronary Chronic Total Occlusion Interventions: A Weighted Meta-Analysis of 18,061 Patients From 65 Studies. J Am Coll Cardiol Intv. 2013;6(2):128-136. 